\title{
Valoración histórica y patrimonial de las ciudades embrionarias: el caso de Mexicali, México
}

Historical and Heritage Valuation of Embryonic Cities: The Case of Mexicali, Mexico Avaliação histórica e patrimonial de cidades embrionárias: o caso de Mexicali, México

Daniel Olvera-García* Berenice Vizcarra*

Recibido: 20 de noviembre de 2020

Aprobado: 29 de junio de 2021

https://doi.org/10.12804/revistas.urosario.edu.co/territorios/a.9956

Para citar este artículo

Olvera-García, D., \& Vizcarra, B. (2021). Valoración histórica y patrimonial de las ciudades embrionarias: el caso de Mexicali, México. Territorios, (45), 109-126. https://doi.org/10.12804/revistas.urosario. edu.co/territorios/a.9956

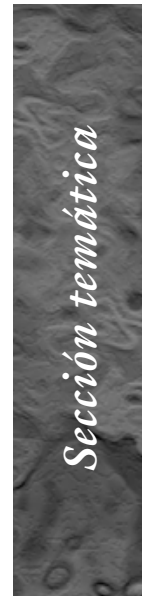

\footnotetext{
* Universidad Autónoma de Baja California (UABC). Correo electrónico:daniel.olvera@uabc. edu.mx. ORCID: https:// orcid.org/0000-00029293-7604

** Universidad Autónoma de Baja California (UABC). Correo electrónico:vizcarra.luz@uabc. edu.mx. ORCID: https:// orcid.org/0000-00025984-0755
} 
Palabras clave

Valoración histórica; patrimonial; arquitectura;

urbanismo; ciudades embrionarias; frontera.

Keywords

Historical assessment; patrimonial; architecture; urbanism; embryonic cities; border.

Palavras-chave

Avaliação histórica; patrimonial; arquitetura; urbanismo; cidades embrionárias; fronteira.

territarias 45
RESUMEN

Dado que muchas de las ciudades de reciente creación han atravesado un proceso de crecimiento y maduración acelerado que, en algunos casos, no ha permitido una documentación adecuada lo que, a su vez, ha provocado que elementos básicos de su evolución se vean perdidos; el trabajo que aquí se presenta tiene el objetivo de elaborar un marco teórico de investigación basada en la revisión bibliográfica del desarrollo urbano-arquitectónico del cuadro histórico de una de estas ciudades embrionarias: Mexicali, México, la cual está conformada por tres sectores que, a su vez, representan la propia estructura de la mancha urbana; es decir, al centro las actividades mixtas, al oriente la vivienda residencial y al poniente los asentamientos populares. Este planteamiento tiene la finalidad de exponer los aspectos fundamentales que caracterizaron este centro fundacional, entender tanto sus cambios como su deterioro e identificar las potencialidades para su valoración histórica y patrimonial.

\section{ABSTRACT}

Given that many of the recently created cities have undergone a process of accelerated growth and maturation that, in some cases, has not allowed adequate documentation, which, in turn, has caused basic elements of their evolution to be lost; The work presented here has the objective of elaborating a theoretical research framework based on the bibliographic review of the urban-architectural development of the historical picture of one of these embryonic cities: Mexicali, Mexico, which is made up of three sectors that, in turn, they represent the very structure of the urban area; that is, to the center, mixed activities, to east, residential housing, and to west, popular settlements. This approach is intended to expose the fundamental aspects that characterized this founding center, understand both its changes and its deterioration and identify the potential for its historical and patrimonial valuation.

\section{RESUMO}

Dado que muitas das cidades recém-criadas passaram por um processo de acelerado crescimento e maturação que, em alguns casos, não permitiu a documentação adequada, o que, por sua vez, fez com que se perdessem elementos básicos da sua evolução; o trabalho aqui apresentado tem o objetivo de elaborar um referencial teórico de pesquisa a partir da revisão bibliográfica do desenvolvimento urbano-arquitetônico do quadro histórico de uma dessas cidades embrionárias: Mexicali, no México, que é composta por três setores que, por sua vez representam a própria estrutura da área urbana; isto é, ao centro, atividades mistas, ao leste, habitações residenciais, e ao oeste, assentamentos populares. Esta abordagem tem por objetivo expor os aspectos fundamentais que caracterizam este centro fundador, compreendendo tanto as suas mudanças como a sua degradação e identificando as potencialidades para a sua valorização histórica e patrimonial. 


\section{Introducción}

El bagaje urbano, arquitectónico y cultural de las ciudades latinoamericanas llega a remontarse a fechas anteriores a la conquista europea de las tierras continentales y caribeñas; asentamientos centenarios que, a partir de la colonización, comenzaron el desarrollo y expansión que hoy las convierte en ciudades patrimoniales tan ampliamente documentadas. Sin embargo, existen territorios que en su momento fueron hostiles para explorar y colonizar pero que en épocas más recientes han sido el paisaje que alberga nuevas civilizaciones. Este es el caso de Baja California, al noroeste de México, tierra abrupta pero fértil cuya capital, nombrada Mexicali, fue establecida casi de manera espontánea hace poco más de cien años (a principios del siglo $\mathrm{xx}$ ) por necesidades externas a las nacionales que, en contraste con otras capitales mexicanas como Guadalajara, Mérida o la Ciudad de México, resulta un organismo incipiente y aún en proceso de configuración urbana, es decir, una ciudad embrionaria.

Puesto que muchas de las ciudades de reciente creación han atravesado un proceso de crecimiento y maduración acelerado que no ha permitido una documentación adecuada, en algunos casos, y lo que, a la vez, ha provocado que elementos básicos de su evolución se vean perdidos; el siguiente escrito se propone elaborar una marco teórico de investigación basada en la revisión bibliográfica del desarrollo urbano-arquitectónico del cuadro histórico de una de estas ciudades embrionarias: Mexicali, México, misma que se conforma por tres sectores que representan la propia estructura de la mancha urbana: al centro las actividades mixtas, al oriente la vivienda residencial y al poniente los asentamientos populares. Este planteamiento tiene la finalidad de exponer los aspectos fundamentales que caracterizaron este centro fundacional, entender tanto sus cambios como su deterioro e identificar las potencialidades para su valoración histórica y patrimonial.

Pareciese que la longevidad es el factor más importante al momento de reconocer el valor histórico y patrimonial de una ciudad, al menos esa ha sido la pauta para el estudio, el registro y la distinción de la gran mayoría de las investigaciones en este rubro. Sin embargo, en tanto que el patrimonio sea entendido como una herencia cultural, reconocida por la sociedad que lo crea y con el fin de fortalecer las identidades presentes en una comunidad multicultural, las ciudades embrionarias también podrán ser consideradas por las aportaciones y testimonios que llegan a esta veloz y cambiante contemporaneidad. Por lo anterior, el principal aporte de la presente investigación es proponer nuevos conceptos y establecerlos como puntos de partida para la generación de conocimiento que, por un lado, expondrán el caso particular del noroeste mexicano y, por el otro, fomentarán la creación de un marco teórico para la valoración histórica territarias 45 
"El término "cimarrón" tiene dos connotaciones en este texto. Primero, su definición textual como adjetivo de un animal/ persona doméstico/a que huye para ser salvaje. Segundo, hace referencia al Ovis canadensis o borrego cimarrón, especie caracteristica y simbólica de la región de Baja California, México.

\section{territarias 45} 112 y patrimonial de otras ciudades embrionarias en Latinoamérica.

Puesto que se espera que este documento sea el primero de una serie de investigaciones urbanas que analicen la evolución y crecimiento de las ciudades embrionarias con el fin de reconocer su valor histórico, así como sus potencialidades en el ámbito patrimonial, la estructura del escrito se compone de cinco grandes temas: un marco histórico, que expone las primicias en el establecimiento de una ciudad durante el siglo Xx; un marco teóricoconceptual, que sustenta la necesidad de una valorización en términos históricos en las ciudades de reciente creación; el reconocimiento del caso de estudio, que establece una descripción acerca de la organización, particularidades y diferenciaciones urbano-arquitectónicas; la revisión bibliográfica y de archivo, que brinda parámetros para el análisis y contribuye al fortalecimiento de la historiografía urbano-arquitectónica; y la identificación de elementos con valor patrimonial, que presenta los resultados obtenidos el área a modo de conclusión.

\section{Planteamento histórico: Mexicali, tierra cimarrona ${ }^{1}$}

Durante el siglo XVI, al tiempo que Hernán Cortés conquistaba el "cuerno de la abundancia” de América, los cinco grupos nativos que conforman la familia Yumana (Cucapá, Kiliwa, Kumiai, Cochimí y Pai-pai) dominaban las tierras a las orillas del río Colorado, mismas que pronto el propio Cortés bautizaría como la península de California. Mientras la expansión colonial avanzaba a gran velocidad con la fundación de ciudades patrimoniales como Guadalajara y Mérida en 1542, apenas 21 años después de la caída de la gran Tenochtitlan, las californias continuaban siendo inexploradas. Es posterior a 1552-55 y las expediciones de Diego de Becerra, Hernando de Grijalva y Fortún Jiménez Bertandoña, por órdenes del gran conquistador, cuando se navega por las costas de este desértico territorio (Herrera, 2007).

Por siglos la península de California fue un territorio inhóspito, un tanto por su lejanía con los centros de poder - como la ciudad de México-y otro más por las peculiaridades de su geografía. Los primeros intentos de asentamiento fueron, más que infructíferos, un fracaso que concluía en la inanición, deshidratación y enfermedad de los aventureros. El propio nombre de "California", desde un comienzo sugería una tierra insólita, un paraíso terrenal regido por amazonas que, entre otras cosas, habían dominado a las fieras salvajes que ahí habitaban, esto según el famoso escrito de "Las sergas de Esplendían".

Si bien en 1697 el padre jesuita Juan María de Salvatierra funda la misión de Nuestra Señora de Loreto (hoy perteneciente al territorio de Baja California Sur), la primera de una larga cadena de asentamientos misionales; es hasta 1772 
cuando se establece un orden geomorfológico al firmase

el concordato entre los misioneros franciscanos y dominicos para que ambos pudieran realizar su misión evangélica en California, el virrey Bucareli autoriza dividirla en dos partes, la Alta o Nueva California para los franciscanos y la Vieja California para los dominicos. (Instituto Nacional para el Federalismo y Desarrollo Municipal INAFED, 2010, párr. 8)

Posteriormente, esta división política se verá reflejada en el Tratado de Paz Guadalupe-Hidalgo que en 1848 sede la Alta California a los Estados Unidos de América (EUA). Para ese momento, la fundación de asentamientos misionales había sido pausada con la Ley de Secularización de 1833. Nótese que, en contraste con las ciudades coloniales antes mencionadas, trazadas a partir de los tratados de urbanismo barroco, vestidas con edificios de estilo neoclásico y respaldadas por 300 años de cultura (para ese entonces), en las californias se perpetuaba el austero orden misional tanto en lo político y cultural, como en lo arquitectónico y las pequeñas concentraciones de población que conformarían lo urbano.

Así, en tanto que la Ciudad de México recibía celebres arquitectos europeos, como el francés Émile Bernard o el italiano Adamo Boari, con el afán de "afrancesar" las calles mediante proyectos cumbre como el Palacio Legislativo o el Palacio de
Bellas Artes (1904-1934), respectivamente; el naciente poblado de Mexicali emergía entre simples ramadas y complejas construcciones de adobe, madera de pino y concreto; que al poco contaría con la influencia de la arquitectura, el urbanismo y la ingeniería estadounidense, sumado de la mano de obra y la experiencia constructiva de los migrantes que provenían de otras entidades mexicanas.

Por todo lo anterior, la domesticación de estas tierras en realidad se hizo posible hasta finales del siglo XIX, cuando empresarios e inversionistas norteamericanos se valieron de los avances tecnológicos, consecuentes de la revolución industrial, y el fructuoso capital que generaban ciudades en auge, como lo fue Chicago (lugar de donde provenían), para comenzar con una serie de proyectos hídricos que concluirían con la conformación de fructuosos valles agrícolas irrigados por las aguas del río Colorado. Esta labor sin precedentes formaba parte de los proyectos de inversión extranjera fomentados por el gobierno de Porfirio Diaz para la industrialización y modernización del país, que se concretaron a partir de la Ley de deslinde y colonización de terrenos baldíos de 1883 .

Así comienza la urbanización en Baja California, con el establecimiento de ciudades como Ensenada en 1882, Tijuana en 1889 y Mexicali en 1903. Esta última, será nombrada capital del territorio a partir de 1915 y resultará el caso a estudiar en este escrito, puesto que su territarias 45 
relativa juventud la coloca en una posición divergente con respecto a la conservación patrimonial. Por un lado, dada su conformación en el siglo $\mathrm{Xx}$, las leyes de conservación patrimonial mexicanas no la ubican en posibilidad de resguardo; por otro lado, las particularidades en su gestación le brindan una peculiar riqueza en espera de ser registrada.

Mexicali es una ciudad embrionaria que guarda elementos culturales, arquitectónicos y urbanos privativos que requieren ser estudiados y documentados ante la veloz evolución urbana y, con ello, la posible pérdida de un patrimonio singular. Elementos culturales que son producto de los numerosos movimientos migratorios comprendidos desde inicios del siglo xx hasta la actualidad y que abarcan pobladores de origen chino, hindú, ruso, norteamericanos, centroamericanos, afrodescendientes, así como compatriotas del centro y sur de México; elementos arquitectónicos, por la cercanía con los materiales prefabricados provenientes de EUA, la mano de obra con experiencia en técnicas constructivas mexicanas, la tendencia a la tipología clásica en las construcciones de la época, así como las especificaciones climáticas y estructurales de una región árida y sísmica, elementos que se reflejan en el estilo californiano o como le especifican Robles Cairo \& Calderón Aguilera (2018a) un neocolonial californiano; y los elementos urbanos que, similar a la mezcla arquitectónica, conjugan teorías de urbanización norteamericanas, proyectos y gestión de política nacional, límites geopolíticos y topográficos, además de una organización y ordenamiento territorial basado en la producción económica, derivan en aquello conocido como ciudad espejo (figura 1).

Es así como el argumento principal de este escrito señala que, más allá de la longevidad como factor único de la valoración histórica y patrimonial, es necesario considerar los elementos que fortalecen las identidades en esta cambiante contemporaneidad. La multiculturalidad que se expresa en el centro fundacional de Mexicali, Baja California, merece ser objeto de estudio en el marco de un foro sobre lecturas históricas del territorio y del patrimonio.

\section{Descripción de la estructura urbana: definición del centro fundacional de Mexicali}

Si bien Mexicali tiene como fecha de establecimiento el año de 1903, la traza urbana de su centro histórico es posterior a 1907, ya que el primer conjunto de edificaciones fue arrasado apenas dos años después de la fundación por el desborde del canal Álamo, uno de los ramales del río Colorado derivado del encausamiento de sus aguas para la irrigación agrícola.

Esta cuadrícula llamada Primera Sección, fue diseñada en conjunto con el centro histórico de la ciudad hermana de Calexico, California, por el ingeniero norteamericano Charles Rockwood. 
Figura 1. Mapa de las ciudades espejo Mexicali-Calexico

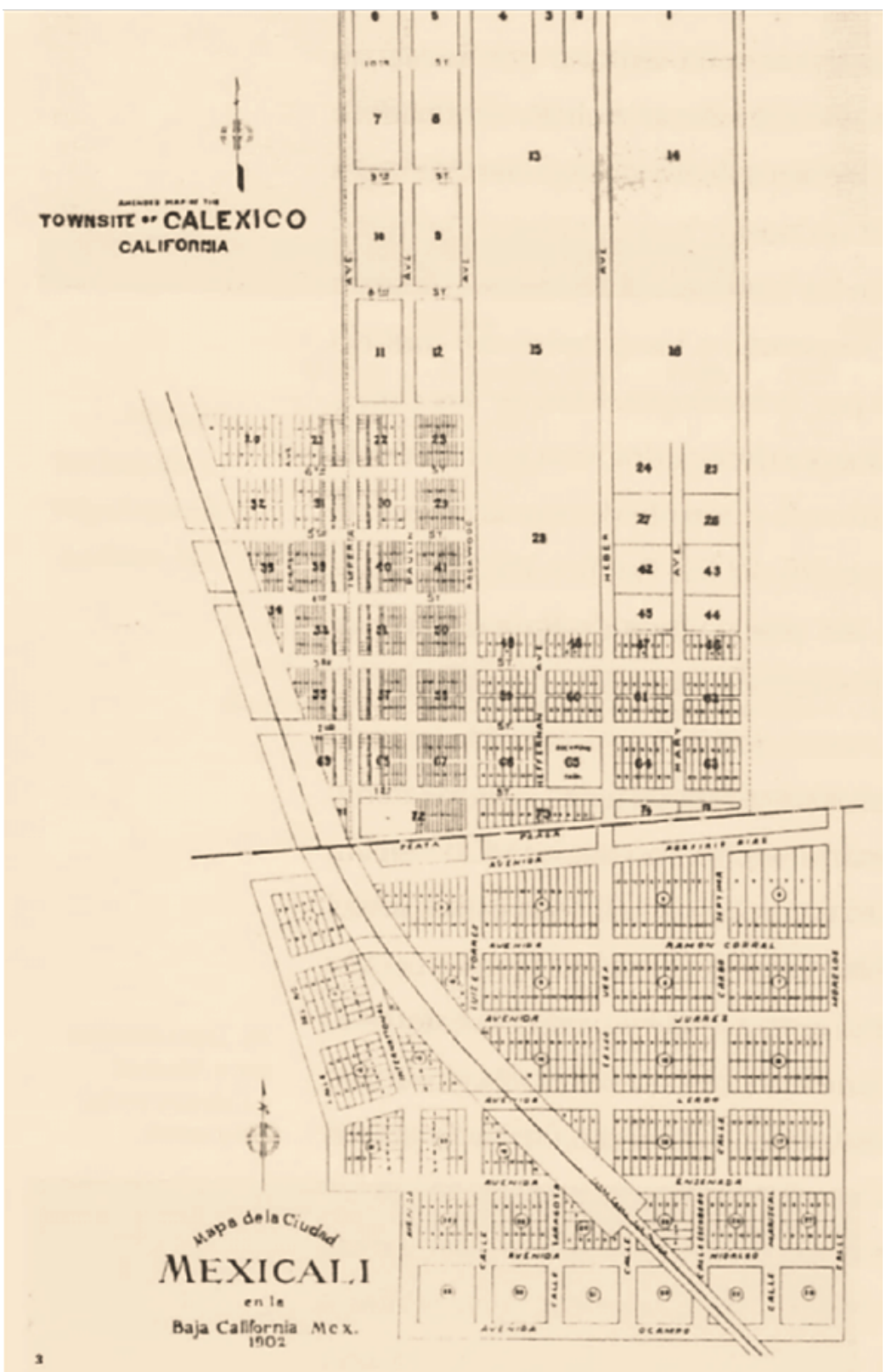

Fuente: archivo histórico de Mexicali, s/f.

territarias 45 115

VALORACIÓN HISTÓRICA Y PATRIMONIAL DE LAS CIUDADES EMBRIONARIAS 
Su morfología se caracteriza por las amplias manzanas urbanas rectangulares, divididas por callejones de servicio, que siguen la orientación diagonal de la propia línea fronteriza con EUA. La ortogonalidad que brinda a primera impresión se rompe con la transversal línea del ferrocarril y el barranco que genera el cuerpo de agua nombrado Río Nuevo, cicatriz que dejaron las inundaciones de 1905 y que desde entonces marcó la frontera poniente de esta primigenia colonia (figura 2 ).

En la Primera Sección se establecieron los iniciales comercios y edificios públicos. Hoy en día, se conservan el parque Niños
Héroes de Chapultepec, inaugurado en 1915; la antigua escuela Cuauhtémoc del mismo año (Robles Cairo, 2009); la plaza Santa Cecilia, nombrada en honor a la patrona de los músicos, quienes se reúnen aquí; la catedral de Nuestra Señora de Guadalupe, edificación de 1940; además de la legendaria "Chinesca", barrio con escondrijos entre callejones y túneles habitados por la numerosa comunidad china en la ciudad.

Al oriente de la primera se instala la Segunda Sección, 40 manzanas urbanas que prolongan las características de la traza, orientación, dimensiones y

Figura 2. Secciones de Mexicali en 1928

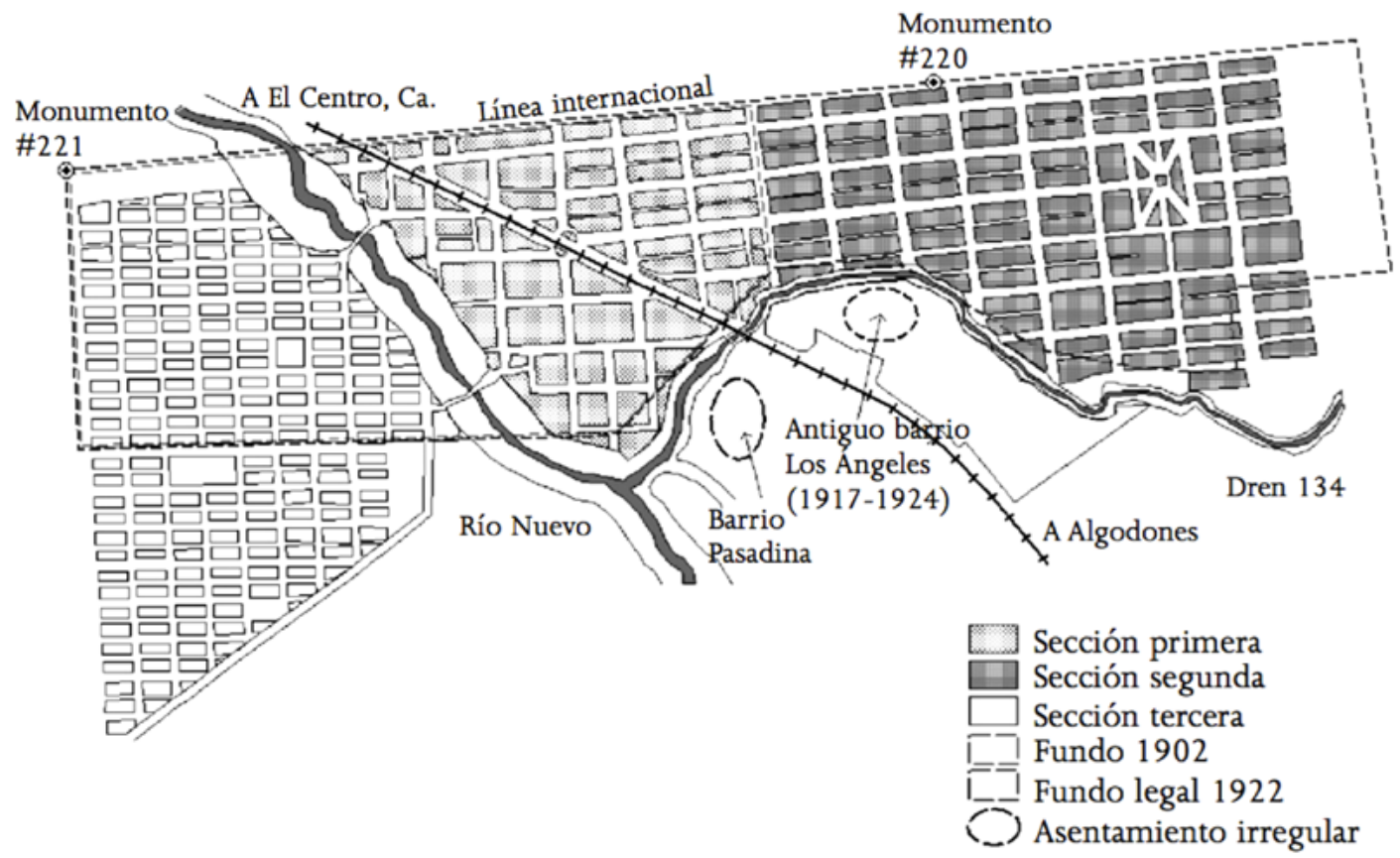

territarias 45

Fuente: Álvarez de la Torre, 2004. 
organización de la sección anterior. Su distinción la otorga el bulevar Álvaro Obregón, eje longitudinal que, a lo largo de dos kilómetros, define el propósito de la colonia: un emplazamiento de los espacios de poder políticos y económicos. Por ello, es aquí donde es posible encontrar a la mayoría de las edificaciones con valor patrimonial de la ciudad.

El antiguo Palacio de Gobierno, inaugurado en las fiestas patrias de 1922, es uno de los edificios más destacados de la Segunda Sección, debido a su hermosa fachada de tipología clásica que funge como remate visual del bulevar Álvaro Obregón. Por su buen estado de conservación, también sobresalen la escuela Leona Vicario y la antigua compañía Colorado Riverland, ambas de 1924. Un tanto remodelados, pero conservando gran parte de su esencia, se ubican también el antiguo Palacio Municipal y la primera estación de bomberos de la ciudad (González González \& Robles Cairo, 2009).

De tipología funcionalista, se encuentran el antiguo Mercado Municipal de 1955 y la Clínica 31 del Instituto Mexicano del Seguro Social (IMSS) de 1961, cuyo diseño se le reconoce a los arquitectos de origen español Imanol Ordorika Bengoechea y Jokin Ordorika (Del Cueto Ruiz-Funes, 2015). Finalmente, la Biblioteca Pública del Estado y el distrito médico (conjunto destinado al turismo médico) se yerguen con un estilo mucho más contemporáneo, de manera que, la Segunda Sección conjuga el pasado y el presente de una ciudad embrionaria. Las construcciones que aquí se ubican son reflejo del auge y prosperidad de cada etapa del crecimiento urbano; situación contraria al envejecimiento de la Tercera Sección.

Pueblo Nuevo es el nombre de la colonia que en su momento constituyó la Tercera Sección de la ciudad. El peculiar cambio en su denominación, a diferencia de las dos primeras secciones, destaca la apropiación de los pobladores sobre el lugar, los cuales eran, en su mayoría, mano de obra migrante. Es así como la Tercera Sección de la ciudad se destinó a la habitabilidad de la pujante clase trabajadora por medio de la vivienda popular.

Ubicada al otro lado del Río Nuevo, distante de la Segunda Sección y conectada a la Primera únicamente por puentes que como brazos se ensanchan sobre el famoso barranco, Pueblo Nuevo se conforma por un conjunto de manzanas urbanas cuadrangulares que, en total, semejan la misma extensión que las dos primeras colonias juntas. Es decir que, durante la década de 1920, fecha de su formación, correspondía aproximadamente al $50 \%$ de la mancha urbana (Instituto Municipal de Investigación Planeación Urbana de Mexicali, 2013).

De traza norte-sur, con amplios predios y callejones de servicio, las manzanas urbanas de Pueblo Nuevo albergan viviendas de autoconstrucción; excepto en la avenida Michoacán, que rápidamente se situó como un corredor urbano destinado al comercio y otras edificaciones de uso territarias 45 117 
común. Estas dos simples características son las que, a grandes rasgos, otorgan el valor histórico y patrimonial de la colonia. Por un lado, las construcciones de adobe parado y techos de madera, técnica constructiva - cada vez es menos conoci$\mathrm{da}$ - con grandes beneficios para las características sísmicas y climáticas de la región; y, por otro lado, el auge que tomó su zona comercial que, en su momento, significó un subcentro para la creciente urbanización al poniente, debido a la dificultad de transportase a la Primera Sección.

En la actualidad, tanto la vivienda como el comercio que oferta Pueblo Nuevo ha quedado rebasado por los nuevos fraccionamientos residenciales, corredores urbanos y centros comerciales en la periferia de la ciudad; acercando a este barrio fundacional a un proceso de deterioro y olvido.

Es a través de estas tres secciones que Mexicali ordenó su estructura urbana. Al centro las actividades mixtas se extendieron a lo largo de toda la orilla del barranco del Río Nuevo; al oriente la vivienda residencial se abrió paso de manera que es posible leer el crecimiento de la ciudad por décadas de acuerdo con el establecimiento de cada uno de los fraccionamiento y las tipologías de sus edificaciones: la Colonia Nueva en 1950, la colonia Cuauhtémoc Norte en 1960, la colonia Flores Magón en 1970, el residencial San Pedro en 1980, el residencial Balboa en 1990 y el residencial Montecarlo en el 2000 (Instituto Municipal de Investigación Planeación Urbana de Mexicali, 2013); y al poniente los asentamientos populares, los cuales perpetuaron la traza y principales características presentadas en Pueblo Nuevo, como la vivienda de autoconstrucción y el fuerte sentido de comunidad que siempre lo caracterizó.

\section{Marco de interpretación para la valoración histórica y patrimonial de ciudades embrionarias}

Ya que las condiciones de fundación de la ciudad de Mexicali son, por demás, peculiares, y puesto que la finalidad de este escrito es presentar un marco teórico de investigación basada en la revisión bibliográfica del desarrollo urbano-arquitectónico del cuadro histórico de una ciudad embrionaria, será importante establecer un marco de interpretación por medio de la definición de conceptos relevantes como: ciudad embrionaria y ciudad espejo; así como postulados teóricos y prácticos tales como: el patrimonio como parte de la sustentabilidad y la legislación patrimonial mexicana.

Se entiende aquí como ciudad embrionaria a aquel asentamiento que se mantiene en continuo desarrollo, es decir, una ciudad que, a pesar de contar con una estructura urbana bien configurada, tanto sus centros como sus periferias sufren de un constante proceso de erosión y degradación. Puesto que este escrito es una lectura histórica del territorio y del patrimonio, se insiste en estudiar las ciudades embrionarias debido a la vulnerabilidad 
que estas tienen en cuanto a la pérdida de elementos urbanos y arquitectónicos con valor histórico y patrimonial.

La relativa reciente creación de las poblaciones del estado de Baja California ha dejado fuera de la protección legal mexicana muchas de las expresiones arquitectónicas ocurridas durante el siglo Xx, ya que la legislación solo protege a las creadas antes de dicho siglo. Aun así, debido tal vez al desconocimiento tanto de la población como de los gobernantes, ha habido en las tres principales ciudades del Estado un completo desapego a la valoración patrimonial. Lo anterior se subraya debido a que la misma legislación establece que solo los inmuebles construidos en el siglo XIX tienen valor histórico (Ley federal sobre monumentos y zonas arqueológicos, artisticos e historicos, 1972), lo que deja fuera de manera inmediata todo aquello establecido en la ciudad de Mexicali, pues recordemos que su fecha de fundación se remonta a 1903; así como gran parte de las ciudades de Ensenada y Tijuana, instituidas en 1882 y 1889, respectivamente.

Como se explicó anteriormente, el ingeniero estadounidense Charles Rockwood trazó a principios del siglo XX la cuadrícula de la Primera Sección en Mexicali, Baja California, a la par del centro histórico de la ciudad californiana de Calexico. Entre las calles de estos asentamientos binacionales continúa existiendo una correspondencia recíproca, a pesar de que este trazo se vio modificado del lado mexicano durante las inundaciones de 1906 y 1907. En tal sentido, esta relación profundizó el desarrollo de ambas poblaciones a lo largo de todo el siglo pasado, generando una interrelación que iba de lo social a lo cultural con todo lo que esto representa.

De esta manera, se confirma lo dicho por Carrión: "las ciudades 'gemelas o espejo' no existen por sí mismas, sino por el vínculo con la del 'otro lado', creando una interconexión en los bordes de los Estados" (2016, p. 2). Mientras que Reyes Posadas et al. (2002) explica que ciudades espejo es "un concepto geográfico aplicado a localidades urbanas contiguas, separadas por un límite administrativo nacional y/o internacional, cuyas interrelaciones, económica, política y social presenten un grado de complementariedad" (p. 223).

Por medio de una comparación profunda entre las ciudades de Mexicali y Calexico se puede observar una ambivalencia mediante varios aspectos. El primero es la integración de ambas poblaciones en una simbiosis que hacía parecer que eran una sola población, donde la frontera era un límite solamente administrativo, hasta entrada la década de 1940, cuando se empieza a delimitar de manera física. El segundo es el idioma que significaba una barrera cultural, pero que no fue impedimento para una sólida conexión. Un tercer aspecto es la manera de construir, puesto que en Mexicali surgió una amplia variedad de sistemas constructivos para la territarias 45 119 
edificación de viviendas y edificios públicos que integraba técnicas tradicionales con materiales provenientes de la vecina ciudad. Esta diferencia se marcó particularmente con la legislación estadounidense sobre la construcción de edificios, la cual establecía materiales específicos para los inmuebles habitacionales.

Cabe señalar que los puntos 38, $124 \mathrm{y}$ 125 de la Nueva Agenda Urbana abogan por "aprovechar de forma sostenible el patrimonio natural y cultural, tanto tangible como intangible, en las ciudades y los asentamientos humanos, según proceda, mediante políticas urbanas y territoriales integradas e inversiones adecuadas en los planos nacional, subnacional y local" $(\mathrm{Na}-$ ciones Unidas, 2017, p. 15). Además de promover "el uso innovador y sostenible de monumentos y espacios arquitectónicos con la intención de crear valor por medio de restauraciones y adaptaciones respetuosas" (p. 38).

Ya que México es uno de los países integrantes en el programa "Hábitat III", la valoración de los elementos patrimoniales de las ciudades embrionarias no solo haría posible contar con inmuebles protegidos, sino las ciudades tendrían la responsabilidad de salvaguardarlos.

\section{Revisión bibliográfica del desarrollo urbano-arquitectónico del cuadro histórico de Mexicali}

territarias 45

La identificación, clasificación, valoración $y$ registro de los inmuebles con valor histórico y patrimonial en Mexicali, ha sido un trabajo preliminar más no determinante o concluyente hasta el momento. Esta labor ha sido encabezada principalmente por estudiosos y entusiastas del tema, cuyos productos comienzan a ser eco en las nuevas generaciones quienes demandan que se emplee el inicial pero meticuloso conocimiento sobre el tema en los bienintencionados pero insuficientes proyectos del gobierno local.

Entre muchos otros que se escapan de este escrito, se puede dar crédito al trabajo de Cuauhtémoc Robles, Margarita González, Felipe Güicho, Isabel Verdugo, la Sociedad de Historia: Centenario de Mexicali A. C., la asociación de vecinos Pueblo Nuevo 100 años, la asociación Uno del Shenk y Yolanda Sánchez, por registrar el desarrollo urbano-arquitectónico del cuadro histórico de Mexicali, misma que en los siguientes párrafos se expone.

Dentro del patrimonio arquitectónico, la vivienda está también considerada, ya que es el edificio nuclear de las sociedades. Caniggia \& Maffei (1995) hacen un análisis de las construcciones a través de sus procesos de desarrollo a lo largo del tiempo y establecen como punto de partida la conciencia espontánea del individuo donde, con base en el medio ambiente en el que habita, define el cómo y con qué construir. Así mismo, estos autores establecen la importancia de la relación entre el lugar y tiempo de edificación, este último siempre tendrá una relación 
directa con el área donde se desarrolla, lo que ellos llaman "ineludibles vínculos heredados".

La vivienda durante la primigenia Mexicali se desarrollaba a partir de los materiales de la región y de los sistemas constructivos de la población Cucapá, como se puede observar en imágenes de la época. Posteriormente, el arribo de población proveniente de El Álamo y Real del Castillo (lugares desde donde iniciaron movimientos migratorios debido a que la producción minera se había agotado) (Aguirre, 1987 citado en Robles Cairo, 2009), así como otros pueblos de la zona costera del Pacífico, donde ya se habían desarrollado construcciones de adobe tanto para vivienda como para edificios públicos y que tenían un lenguaje arquitectónico similar a construcciones californianas.

Como testimonio se encuentra la primera fotografía tomada en 1902 con edificaciones a lo largo de la Primera Sección. De esta manera, el desarrollo de la ciudad continuó con esa influencia californiana en su trazo ortogonal, con una cuadrícula definida, amplias calles y callejones de servicio, así como con una arquitectura que va desde los edificios públicos hasta los privados, y que hoy en día es por igual reconocida por su valor histórico, pero abandonada a su suerte al no poder ser resguardada legalmente como patrimonio (figura 3 ).

La incorporación de nueva tecnología en materiales constructivos como el concreto fue un detonante en el rápido desarrollo que tuvo la ciudad en sus primeros veinte años. El ejemplo más importante de esto, y el único que se ha preservado de esa primera época de desarrollo, es la Escuela Cuauhtémoc - hoy Casa de la Cultura Municipalconstruida entre 1915 y 1916 (figura 3). La edificación de este inmueble implicó una relación profunda con los prototipos, lenguajes y sistemas de Estados Unidos, así lo establece Robles Cairo \& Calderón Aguilera (2018b), situación que se acentuaba con el distanciamiento geográfico que existía con el centro de México.

Dicha situación se puede ver repetida en varios edificios que se construyeron en la década de 1920. El más destacado de estos, por sus dimensiones y construcción de concreto armado, es el Palacio de Gobierno, hoy Rectoría de la Universidad Autónoma de Baja California (UABC). Robles, quien se destaca como historiador de la arquitectura de Mexicali, la define como una arquitectura con un lenguaje híbrido influenciada de la vecindad con Estados Unidos y la inmigración de mexicanos venidos de diferentes partes del país (González González \& Robles Cairo, 2009). Este lenguaje híbrido no solo se ve en lo arquitectónico, también en los primeros trazos urbanos y en los sistemas constructivos tradicionales como el adobe, que forman parte de la valoración histórica y patrimonial de la ciudad de Mexicali. Este lenguaje híbrido se extendió asimismo a la industria agrícola, territarias 45

121 
${ }^{2}$ Acuerdo diplomático entre México y EUA de 1941 a 1964, cuando mano de obra mexicana trabajó en los campos agricolas estadounidenses. territarias 45 122

Figura 3. Escuela Cuauhtémoc, inaugurada en 1916

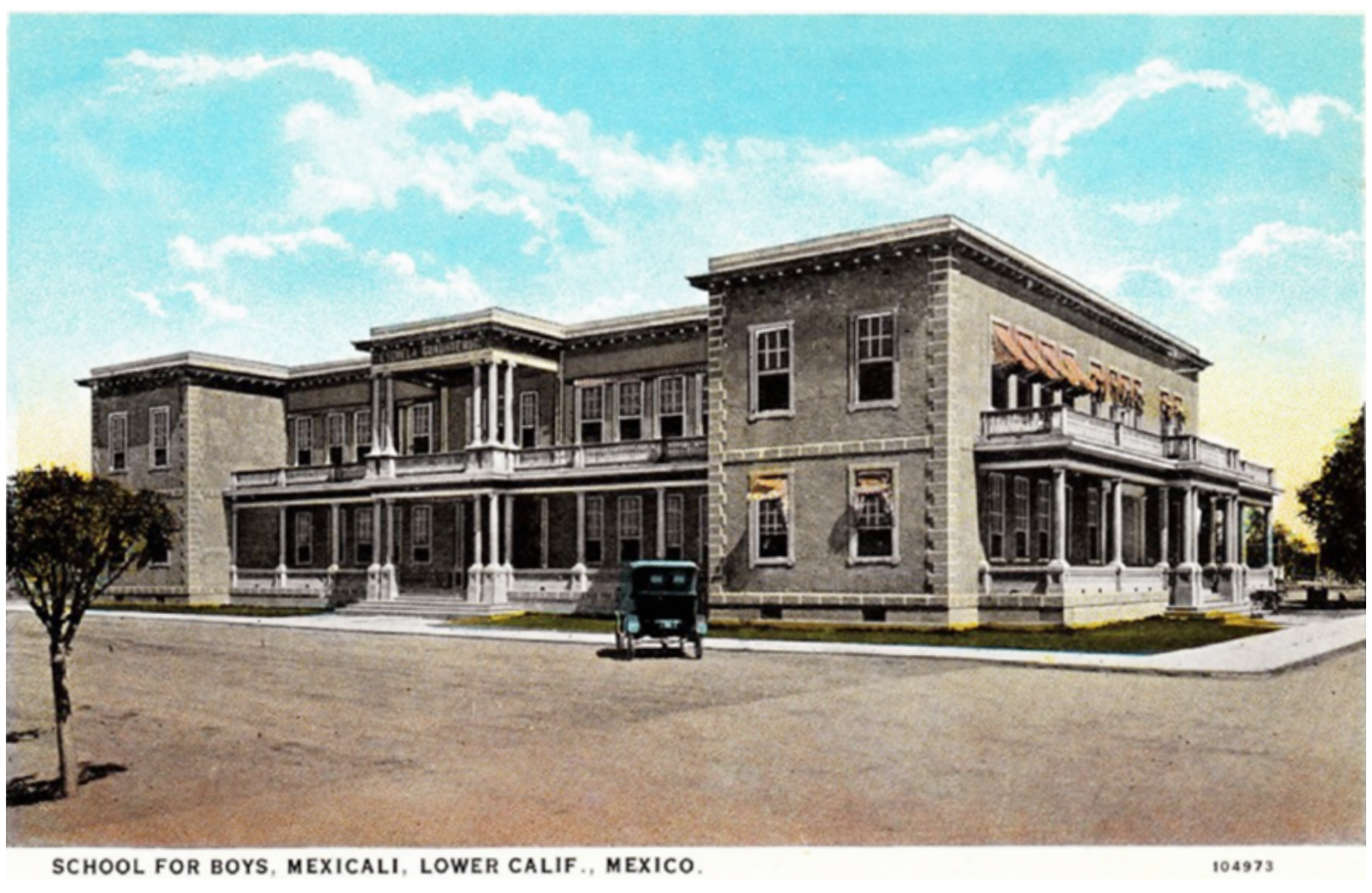

Fuente: archivo histórico de Mexicali, s/f.

principal fuente de ingresos hasta poco más de la mitad del siglo Xx.

En este punto, es importante recordar el origen agrícola de la ciudad ya que, si bien en los años cincuenta la población de Mexicali ya era principalmente urbana, la agricultura era aún la actividad económica primordial de la localidad y de la entidad, lo cual cambia para la década de 1960 con el impulso de la industria maquiladora en las franjas fronterizas (Ley García \& Fimbres Durazo, 2011), debido a la llegada de mano de obra mexicana que regresaba por el fin de la Segunda Guerra Mundial y con ello el llamado Programa Bracero ${ }^{2}$ en 1964, lo que significó un aumento de la población en Mexicali a diferencia de la ciudad de Calexico.

Finalmente, Corona et al. (2014) indican con varios ejemplos la cantidad de patrimonio que existe, no solamente en la ciudad de Mexicali, sino también en la zona agrícola conocida como el Valle de Mexicali, donde resaltan otros tipos de obras con valor patrimonial como las escuelas rurales, las presas, puentes de ferrocarril y sus estaciones, además de compuertas de canales. 
Lo anterior resalta en la actividad que dio vida a la misma ciudad y que no ha podido preservarse, como pueden ser también los ranchos o cascos de hacienda que fungían como primer punto de llegada de los productos agrícolas que se cosechaban y que solo existen en referencias en los libros de historia locales, en los que no se habla de sus características y valor arquitectónico. Como excepción se pude destacar el Rancho Duarte (fundado en 1938) descrito desde una perspectiva histórica por su origen, así como su importancia económica, Peimbert \& Olvera (2018) lo describen como un conjunto similar a los ranchos californios, de función agroindustrial con una arquitectura regional tanto en su diseño como en los materiales constructivos que resalta su valor patrimonial.

\section{Reflexiones finales}

La madrugada del jueves primero de octubre de 2020 se reportó a las autoridades correspondientes el incendio de una vivienda ubicada en la Primera Sección de la ciudad de Mexicali, Baja California. Las notas en los periódicos locales señalan que:

El saldo fue de daños totales y las causas del siniestro no han sido determinadas, pues al ser propiedad particular, el peritaje debe ser solicitado por el propietario [...] De acuerdo con diversos historiadores, en esta casa vivió un tiempo el jefe militar y político del Distrito Norte de Baja California, el coronel Esteban Cantú. (Martínez, 2020)

Así, de un momento a otro un inmueble con valor histórico se vio perdido en una ciudad que continúa tratando de escribir su testimonio en la historia del mundo. Además de atribuírsele una relación con uno de los personajes más importantes para el desarrollo urbano y económico de la Baja California, el coronel Cantú, esta vivienda contaba con elementos interesantes para su estudio, como la estructura de madera, el estilo tipo bungalow y otros detalles arquitectónicos que hoy son cenizas (figura 4).

El coordinador del Archivo Histórico de Mexicali calificó como lamentable la pérdida de esta casa, pues representaba parte de la identidad histórica de los pioneros de esta ciudad. Señaló que esta casa pertenece a la primera etapa del desarrollo urbano de Mexicali, de entre 1915 y 1920, caracterizada por casas de madera y material traído de California. (Martínez, 2020)

A lo largo de este escrito se ha llegado a diversas conclusiones que sustentan la importancia de la valorización histórica de los elementos tangibles aun en las ciudades modernas. Entre otras cosas, se expusieron los aspectos fundamentales que caracterizaron el centro fundacional de un asentamiento de reciente creación como Mexicali, tanto en su obra arquitectónica como en su estructura 
${ }^{3}$ En el reporte independiente de Álvarez, G., Avilés, A. M., Estrella, G., Ortega, G., \& Ranfla, A. (1995), llamado "Alternativas de estrategias para la renovación urbana de Pueblo Nuevo", solicitado por el XIV Ayuntamiento de Mexicali, se señala un total de 14,842 habitantes.

${ }^{4}$ El censo de población $y$ vivienda del 2010, da como resultados absolutos 749,320 habitantes a nivel urbano y 936,826 habitantes en todo el municipio.

territarias 45 124

Figura 4. Vivienda ubicada en la Primera Sección antes de ser incendiada

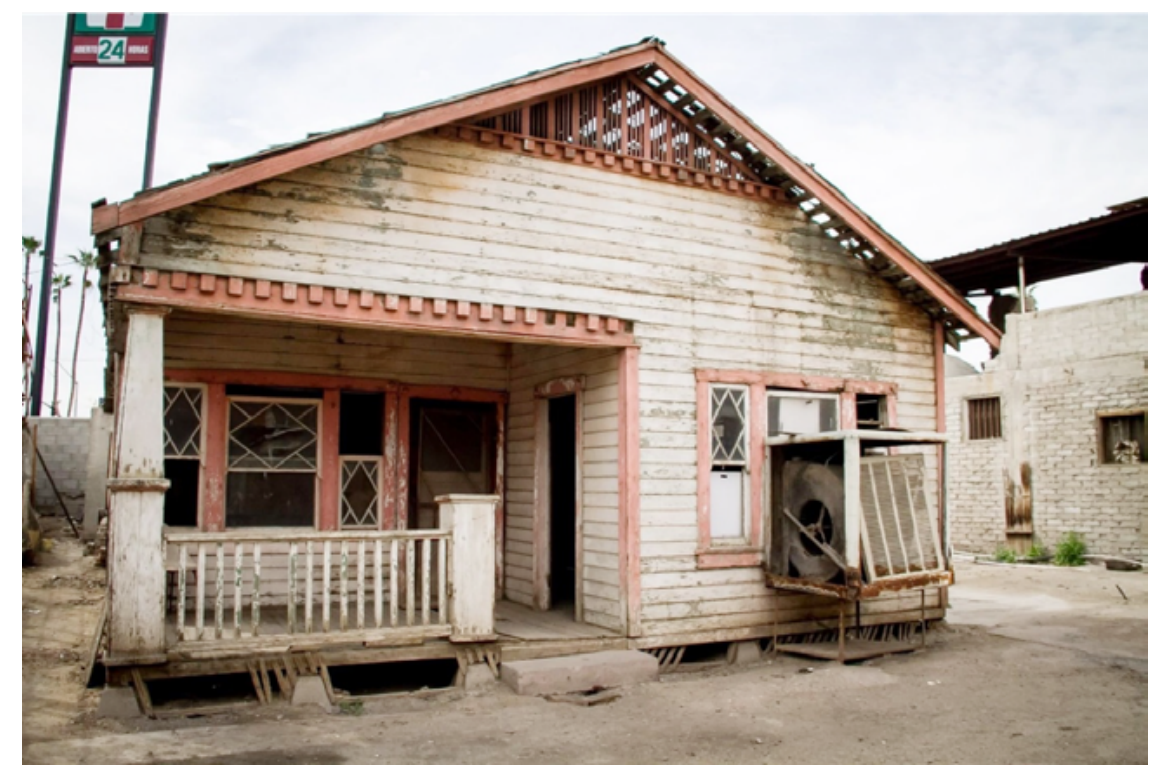

Fuente: Martínez, 2020.

urbana. El veloz y dinámico crecimiento de su población y territorio se evidencia de manera cuantitativa, al pasar de casi 15,000 habitantes en la década de $1930^{3}$ a, aproximadamente, 750,000 habitantes en la mancha urbana en 2010 y cerca de un millón a nivel municipal. ${ }^{4}$ Y también se muestra de manera cualitativa puesto que este acelerado auge ha impedido, en consecuencia, la definición de elementos patrimoniales en la región.

Sería significativo, pues, que la legislación mexicana se tomase el tiempo de revisar y analizar la importancia de los elementos patrimoniales en el desarrollo de una cultura contemporánea. En este sentido, aquí se ha presentado una revisión de propuestas que diversos historiadores locales y estudiosos del tema han sugerido como piezas tangibles en la cultura del noroeste de México. A nivel urbano se puede hablar del barrio de la Chinesca y el barrio de Pueblo Nuevo como sectores que deberían ser integrados en la definición del centro histórico de Mexicali. Mientras que, a nivel arquitectónico, los ejemplos van desde los ranchos en el Valle de Mexicali hasta las viviendas en el sector fundacional de la ciudad; incluyendo también edificios públicos y privados como el mencionado antiguo Palacio de Gobierno y la Escuela Cuauhtémoc, herencias culturales que merecen ser valoradas. 


\section{Referencias}

Álvarez de la Torre, G. B. (2004). Los actores en el desarrollo urbano de Mexicali: 1903-1928. SINER, 2(39), 7-22.

Caniggia, G., \& Maffei, G. L. (1995). Tipologia de la edificación: estructura del espacio antrópico. Celeste.

Carrión, F. (2016, 17 de febrero). Ciudades de frontera en América Latina. El Pais. https://elpais.com/ elpais/2016/02/17/seres_urbanos/1455692400_145569.html

Corona, E. A., Arredondo, J. A., Rojas, R. I., \& Martínez, F. A. (2014). Mexicali una ciudad sin valoración histórica de su patrimonio cultural (pp. 814-838). Ponencia en la Conferencia Internacional Ciudad Virtual y Territorio. IX Congreso Ciudad y Territorio Virtual, Roma. http://upcommons.upc.edu/ revistes/handle/2099/16303

Del Cueto Ruiz-Funes, J. I. (2015). Presencia del exilio español en la arquitectura mexicana. Facultad de Arquitectura, Universidad Nacional Autónoma de México, unam.

González González, A. M., \& Robles Cairo, C. (2009). Arquitectura histórica de Mexicali: escuelas y edificios públicos: Universidad Autónoma de Baja California, UABC.

Herrera, Pablo. (2007). Fray Junipero Serra: civilizador de las Californias. Universidad Autónoma de Baja California, UABC.
Instituto Municipal de Investigación Planeación Urbana de Mexicali. (2013). Crecimiento histórico de la Ciudad de Mexicali (1903 - 2013). http:// www.mexicali.gob.mx/sitioimip/ fotos/3fc6284dedb3.jpg

Instituto Nacional para el Federalismo y Desarrollo Municipal (INAFED). (2010). Enciclopedia de Los Municipios y Delegaciones de México. http:// inafed.gob.mx/work/enciclopedia/ EMM02bajacalifornia/historia.html Ley federal sobre monumentos y zonas arqueológicos, artísticos e históricos. (1972). Cámara de Diputados del H. Congreso de la Unión. DOF 6 de mayo de 1972. http://www.diputados.gob. $\mathrm{mx} /$ LeyesBiblio/pdf/131_160218. pdf

Ley García, J., \& Fimbres Durazo, N. A. (2011, septiembre-diciembre). La expansión de la ciudad de Mexicali: una aproximación desde la visión de sus habitantes. Región y Sociedad, 23(52), 209-238.

Martínez, S. (2020, 02 de octubre). Arde la casa del coronel Esteban Cantú. El Imparcial. https://www.elimparcial.com/mexicali/policiaca/ Arde-la-casa-del-coronel-EstebanCantu-20201001-0008.html

Naciones Unidas. (2017). Nueva agenda urbana [Habitat III]. http://uploads. habitat3.org/hb3/NUA-Spanish. pdf

Peimbert, A., \& Olvera, D. (2018). El rancho Duarte del Valle de Mexicali: 
Arquitectura de la producción del auge algodonero en México. Topofilia, (17), 103-130. http://69.164.202.149/topofilia/index.php/topofilia/article/ view/31

Reyes Posadas, C., Rangel Calvillo, E., Enríquez Denton, F., \& Hernández Figueroa, E. (2002). Explorando la geografía de México 2. Nuevo México.

Robles Cairo, C. (2009). La arquitectura de Mexicali (origenes) (Primera). Universidad Autónoma de Baja California, UABC.

Robles Cairo, C., \& Calderón Aguilera, C. M. (2018a). Arquitectura del siglo
Xx en la frontera norte de México: la arquitectura neocolonial californiana y el patrimonio arquitectónico bajacaliforniano. Mundo, Arquitectura, Diseño Gráfico y Urbanismo, MADGU, 1(2), 1-24. https://doi.org/10.36800/ madgu.vli2.28

Robles Cairo, C., \& Calderón Aguilera, C. M. (2018b). Patrimonio e industrialización. El concreto armado en la arquitectura de Mexicali, México, 1915-1930. Gremium, 5(9), 19-34. https://editorialrestauro.com.mx/ gremium/index.php/gremium/article/view/247 